\title{
LAS PROPUESTAS CURRICULARES EN ESCUELAS DE ELITE EN BUENOS AIRES: DIFERENCIACIÓN INSTITUCIONAL PARA EDUCAR EN EL PRIVILEGIO
}

\author{
Buenos Aires Elite High Schools' Curricular Proposals: Institutional \\ Differentiation to Educate in Privilege
}

\author{
SANDRA ZIEGLER*1 \\ VICTORIA GESSAGHI**2 \\ SEBASTIÁN FUENTES ${ }^{* * * 3}$ \\ *FLACSO/UBA. Argentina. sziegler@flacso.org.ar \\ ${ }^{* *}$ CONICET/UBA/FLACSO. Argentina. victoriagessaghi@hotmail.com \\ ${ }^{* * *}$ CONICET/FLACSO/UNTREF. Argentina. sebasfuentes3@gmail.com
}

Recibido: 23-03-2017

Revisado: 08-05-2017

Aceptado: 09-08-2017

Resumen: Este artículo explora la experiencia escolar en las escuelas de elite de la Argentina. Se analiza el currículum desplegado por las instituciones como propuesta cultural que las escuelas ponen a disposición de las familias. Los resultados se basan en entrevistas en profundidad realizadas en cinco escuelas de la ciudad y la provincia de Buenos Aires, así como en el análisis de documentos y páginas web institucionales. Se concluye que las propuestas curriculares de las escuelas generan experiencias de aprendizaje y de relación que familiarizan a los estudiantes con las posiciones, las estéticas y los "valores" diferenciadores del sector o grupo social al que pertenecen.

Palabras clave: elites; currículum; experiencia; familias.

\begin{abstract}
This paper explores school experience in elite institutions in Argentina. It analyzes the curriculum offered by these schools as cultural proposals available to families. The results are based on in-depth interviews conducted in five schools in the City and Province of Buenos Aires and the analysis of documents and institutional websites. It is concluded that the curriculum these schools highlight allows a series of experiences and relations with knowledge that indicate a commitment to a training that familiarizes students with the distinctive positions, aesthetics and "values" they hold as social group.
\end{abstract}

Keywords: elites; curriculum; experience; families.

\footnotetext{
${ }^{1}$ Doctora en Ciencias Sociales, magíster en Ciencias Sociales con orientación en Educación (FLACSO). Licenciada en Ciencias de la Educación en la Universidad de Buenos Aires (Argentina). Investigadora senior del Programa Educación, Conocimiento y Sociedad de FLACSO Argentina, donde coordina el Núcleo de estudios sobre elites y desigualdades socioeducativas y la Maestría en Ciencias Sociales con orientación en Educación. Profesora adjunta regular en la Facultad de Ciencias Exactas y Naturales de la Universidad de Buenos Aires (Argentina).

2 Doctora en Antropología Social en la Universidad de Buenos Aires (Argentina) y magíster en Ciencias Sociales con orientación en Educación (FLACSO). Investigadora del CONICET, del Programa de Antropología y Educación de la Universidad de Buenos Aires y del Núcleo de estudios sobre elites y desigualdades socioeducativas de FLACSO. Docente de la Universidad de Buenos Aires y de la Universidad Nacional de La Matanza (Argentina).

${ }^{3}$ Doctor en Antropología Social de la Universidad Nacional de San Martín (Argentina), magíster en Ciencias Sociales con orientación en Educación (FLACSO). Licenciado en Filosofía. Investigador del CONICET, integra el Núcleo de estudios sobre elites y desigualdades socioeducativas de FLACSO. Profesor-investigador de la Universidad Tres de Febrero (Argentina). Investigador del Proyecto GLOBALSPORT/Universidad de Ámsterdam, financiado por el European Research Council (ERC).
} 


\section{LOS ESTUDIOS SOBRE EDUCACIÓN DE LAS ELITES: ¿QUÉ ENSEÑAN LAS ESCUELAS?}

El estudio de la formación de las elites ${ }^{1}$ floreció en la Argentina con el inicio del siglo XXI. Los trabajos gestados al calor dela crisis socioeconómica de ese momento eran un campo novedoso de análisis dentro de una sociología de la educación inquieta ante la evidencia de un sistema educativo fragmentado. Sin embargo, esta preocupación contaba con una errática tradición dentro de la sociología argentina. En el texto inaugural Los que mandan (1964), José Luis De Imaz analiza la trayectoria de quienes ocupaban en aquel momento las más altas posiciones institucionalizadas y plantea que, a diferencia de otros países, la elite vernácula no contaba con un circuito formativo institucionalizado. Aunque no fue el único en aquel contexto, constituyó uno de los primeros enfoques que asoció la posición social destacada con la presencia o ausencia de determinados circuitos formativos y se volvió de referencia para los desarrollos que treinta años más tarde se abocaron a estudiar las trayectorias formativas de las elites en Argentina (Heredia, 2012; Canelo, 2012; Sarrabayrouse, 1999, entre otros).

Si hace unos años no dejábamos de remarcar lo novedoso y necesario de este impulso por estudiar las prácticas educativas de los sectores privilegiados de la sociedad, hoy es posible reconocer una diversidad de investigaciones que se han ocupado de las elites y de sus experiencias formativas (Méndez, 2013; Gessaghi, 2016; Ziegler; 2015; Fuentes, 2012 y 2015b; Rodríguez Moyano, 2012; Dallaglio,2015; Villa, 2012; Goenaga, 2016; Fabrizio y Montero, 2014). Desde una perspectiva crítica, estos análisis han atendido a los procesos de formación de los sectores dirigentes o de las elites, documentaron las relaciones entre familias, escuelas y universidades, describieron sus circuitos de sociabilidad y educativos, y analizaron sus prácticas educativas en articulación con el sistema educativo argentino. Estos estudios recuperaron los aportes de la historia y la sociología de la educación en la Argentina que desde la década de 1980 permitieron comprender el carácter elitista que algunas escuelas y modalidades tuvieron en el desarrollo del sistema educativo argentino (Tedesco, 1986; Puiggróss, 1990; Dussel, 1997). Asimismo, se inscriben en la tradición iniciada por los trabajos de Braslavsky (1985), Kessler (2002) y Tiramonti (2004), que echaron luz sobre los modos en que las prácticas de distintos grupos sociales mediadas y moldeadas a su vez por las prácticas estatales- configuran circuitos escolares diferenciados.

Estas investigaciones han construido un corpus de constataciones convergentes que permiten poner en cuestión la promesa que el sistema educativo argentino formuló

\footnotetext{
${ }^{1}$ Llamamos elites a aquellos sujetos que detentan posiciones sociales de privilegio alcanzadas en base al volumen, la trayectoria y el tipo de capital que poseen (ya sea económico, cultural o social en diferentes combinaciones) (Bourdieu, 1984).
} 
históricamente, sobre todo para el nivel primario: la escolarización es garante de igualdad de oportunidades y de movilidad social para todos los ciudadanos. Sucintamente, la crítica se concentra en la constatación de la existencia de escuelas muy diferenciadas según el origen social de los alumnos que asiste a cada institución. En las últimas décadas, este campo de estudios ha documentado los modos en que la educación asegura privilegios para los hijos de los sectores acomodados, o cómo estos sectores sociales construyen circuitos educativos que la propia configuración del sistema educativo permite o legitima. ${ }^{2}$

En el marco de los procesos de diferenciación institucional y de circuitos indicada, una serie de presupuestos provenientes de los discursos mediáticos y de sentido común han construido una fuerte asociación simbólica entre escuelas de elite, educación de gestión privada y calidad educativa, supuesto que la investigación científica ha comenzado a problematizar (Albornoz, Furman, Podestá, Razquin y Warnes, 2016; Gessaghi, 2016; Cerletti, 2014; Cervini, 2003; Ganimian, 2013; Narodowsky y Moschetti, 2015; Rivas, 2010; Santillán, 2012).A partir de la información recabada por los exámenes PISA Argentina en sus ondas 2009 y 2012, Albornoz et al. (2016) señalan que el desempeño diferencial de la escuela privada se explica por diferencias en el entorno social ${ }^{3}$ de la matrícula que permanecen inalteradas en el período analizado. Otros trabajos (Sacerdote, 2011) subrayan que el mayor nivel socioeconómico del alumnado y el efecto pares ${ }^{4}$ es lo que incide en los mejores resultados que obtienen las instituciones de gestión privada en nuestro país.

En el mismo sentido, los trabajos sobre trayectorias educativas de los sectores privilegiados (Ziegler, 2007; Gessaghi, 2016; Fuentes, 2015b; Luci, 2012; Villa, 2012) complejizan la vinculación entre "calidad educativa" y escuelas de elite. Sin pretender resolver esas asociaciones, registran que la calidad educativa en este tipo de escuelas no se reduce a lo que allí se enseña, sino a las condiciones que configuran la experiencia de socialización que propician, al tipo de relaciones y lazos sociales que se construyen. Las estrategias de socialización entre iguales son una marca de una educación que se concibe como de calidad en función del origen social de los alumnos y de las apuestas por el destino de ellos. Sin embargo, aquello que estas escuelas se

2 En cada "fragmento" del sistema se ponen en juego la posición socioeconómica y la configuración de valores con los que se estructuran los diferentes grupos sociales y desde las cuales cada familia selecciona la escuela. Algunos estudios han documentado que las estrategias familiares e institucionales varían de acuerdo con los distintos fragmentos de elites (Ziegler, 2004; Gessaghi, 2013; Méndez, 2013; Rodríguez Moyano, 2012; Villa, 2005). Existen contribuciones diferenciales de las escuelas que se constituyen en base a la interpretación que estas y sus destinatarios realizan en torno a los requisitos necesarios para consolidar las posiciones de privilegio (Ziegler, 2007).

3 Los estudios muestran que el sector atiende a todos los niveles socioeconómicos. Sin embargo, entre los sectores más ricos de la población la elección de la escuela privada es significativamente más elevada que la estatal (Albornoz et al., 2016).

4 Se refiere a la posible relación entre el rendimiento de un alumno y las características o comportamiento de sus pares. 
proponen enseñar, el análisis del currículum en sentido amplio, constituye un área de vacancia hasta la actualidad.

A los fines de contribuir a ese campo inexplorado por la sociología de la educación y de las elites y con la voluntad de abrir una discusión que permita afinar nuestras interpretaciones respecto de la desigualdad educativa en la Argentina, este artículo explora los contenidos de la experiencia escolar de los alumnos en cinco escuelas secundarias de elite de la Ciudad y la Provincia de Buenos Aires. El nivel secundario de educación en la Argentina goza de una historia particular y un presente problemático. Proyectada en sus orígenes como institución formadora de elites y de sectores medios ilustrados, se halla tensionada actualmente por un proceso de masificación -que acontece sin cesar desde la década del 50- y una serie de políticas que tramitan su obligatoriedad para la población joven, al mismo tiempo que conserva un formato pensado para la selección social. Nuestra mirada puesta en las escuelas de elite tiene como trasfondo la manera en que se transmite el privilegio en contextos de ampliación del derecho a la educación.

El tipo de análisis que proponemos se basa, primero, en una concepción amplia del currículum. Nos interesa interrogar las propuestas de enseñanza de las escuelas como la propuesta cultural que ponen a disposición de sus alumnos y entenderlas en el marco de las relaciones entre familias y escuelas, entre escuelas que compiten diferenciándose entre sí, o que compiten imitando y construyendo propuestas cuyos destinatarios son interpelados en función de intereses, apuestas, ideologías e historias en común. La pregunta sobre el tipo de experiencia formativa que buscan las escuelas de elite se inserta en una problemática más general, que es la relación de los actores con los saberes, las prácticas y los consumos culturales en la configuración desigual de la sociedad argentina, que traza fronteras entre sectores sociales.

Tal como vienen indicando algunas investigaciones (Ziegler, 2017; Kahn, 2011), las escuelas que se presentan como formadoras de elite aspiran a promover en sus estudiantes una sensibilidad, una estructura afectiva y estética sobre el privilegio. El currículum y la propuesta de enseñanza están vinculados con esa experiencia, aunque no la explican en su totalidad. Nuestro aporte se enfoca en el análisis de la propuesta de enseñanza y de algunos elementos de la experiencia producida entre los estudiantes.

\section{LAS CARACTERÍSTICAS DEL TRABAJO DE CAMPO}

El trabajo de campo realizado involucra entrevistas en profundidad a 50 padres/madres, 20 profesores o directores de 5 escuelas secundarias de la Ciudad y la Provincia de Buenos Aires, así como la lectura, recopilación y análisis sistemático de documentación institucional y de las páginas web de las instituciones elegidas. Dichas páginas constituyen dispositivos que tejen relaciones en el espacio social en el que son producidos: seleccionando contenidos de la vida escolar mediados por las prácticas de 
uso y las condiciones de producción y reconocimiento específicas del soporte tecnológico, establecen una relación entre lo que la institución quiere y puede comunicar sobre sí misma y el modo en que dichos discursos moldean a la escuela (Landau, 2011).

Las instituciones fueron elegidas en función de investigaciones previas, que nos acercaron a las escuelas que apuestan o se presentan como formadoras de elites. En esas indagaciones pudimos apreciar cómo estas se abocaban a la formación de los hijos/as de dueños de empresas, de intelectuales y académicos, de administradores estatales, banqueros, profesionales liberales, propietarios de tierras y dueños de empresas y comercios de tamaño medio. Las instituciones elegidas nos permiten mostrar la diversidad de escuelas secundarias que atienden a estos sectores: una de ellas es pública, gestionada por la universidad (en adelante "escuela pública universitaria"); las otras cuatro son privadas. Tres son católicas (en adelante "escuelas católicas" 1, 2 y 3), la cuarta es laica ("escuela privada laica"). Todas son renombradas por su prestigio institucional y gozan de una larga historia. El colegio universitario fue elegido por su proceso de selección del alumnado y su lugar gravitante en la historia de la formación de líderes nacionales. Entendemos que estas escuelas apuestan a la formación para ocupar posiciones de privilegio, en otras palabras: pasan por sus aulas quienes pertenecen o buscan afianzarse entre los grupos más aventajados económica, social y culturalmente.

Puesto que el análisis del currículum oficial en sentido estricto (entendido meramente como plan de estudio declarado) es necesario pero insuficiente para dar cuenta de la diferenciación que opera en las escuelas de elite, hemos sistematizado la información producida sobre el conjunto de la propuesta de enseñanza, organizando el análisis en cinco claves de la diferenciación: 1) las propuestas de plan de estudios; 2)la ubicación y la infraestructura escolar; 3) el tipo de regulación sobre la convivencia, las relaciones e interacciones entre estudiantes; 4) la circulación de los estudiantes y los saberes a nivel nacional y global; y 5) lo que hemos denominado "currículum solidario".

\section{Más allá de la suma: apuestas institucionales en la definición del plan de estudios}

Si bien el currículum común —-prescripto por el Ministerio de Educación de la Nación y las jurisdicciones en donde se localizan los establecimientos- se encuentra presente en casi todas las escuelas abordadas (con excepción de la escuela pública universitaria), éstas desarrollan adicionalmente programas que contribuyen a forjar formas de diferenciación, como actividades extraescolares y programas de exámenes internacionales de inglés. Identificamos una estructura de plan de estudios que concentra los saberes escolares ordenados de acuerdo al currículum disciplinar clásico en base a disciplinas altamente diferenciadas entre sí. En términos de Bernstein (1988) 
presentan un currículum con una clasificación fuerte en tanto las disciplinas están estrictamente delimitadas y compartimentalizadas. Dentro de esta estructura el grado de electividad de los alumnos es prácticamente nulo. Se trata de un plan de estudios clásico de secuencia lineal y disciplinar que no admite la presencia de enfoques interdisciplinarios, ni la opcionalidad para profesores ni estudiantes en términos de la presentación del saber y del recorrido a efectuar. El currículum formalmente se presenta mediante los planes de estudio, pero no contamos con información acerca de las interacciones en el espacio del aula que den cuenta ${ }^{5}$ si en la puesta en acción hay experiencias que modifiquen esta estructura formal.

Esas propuestas se flexibilizan en todas las actividades aledañas al núcleo anteriormente mencionado, que representa el espacio en donde se torna visible la diferenciación del menú de experiencias que estas escuelas plantean. Si otras escuelas parecen haber desarrollado en los últimos años una actualización de sus estrategias pedagógicas orientadas a incorporar "inteligencias múltiples", "habilidades emocionales", "trabajo en equipo" en sus alumnos, o reforzar el acompañamiento pedagógico atento a las diferencias y a favorecer los enfoques "inclusivos de la diversidad", la presentación de las escuelas en general se centra en una formulación conservadora del currículum. Es posible rastrear un énfasis enciclopédico en sus programas de estudio, en donde el eje está colocado en la adquisición de los saberes de las disciplinas. La presencia del humanismo como sustrato cultual de la modernidad que dio sostén a los saberes y conocimientos de dicha época para transformarlos en el contenido del currículum escolar continúa vigente.

La escuela privada laica denota estar desarrollando recientemente un giro en sus enfoques de enseñanza. La presentación de su página web explica la decisión de generar una pedagogía más centrada en los aprendizajes de los alumnos —más que en la enseñanza enfocada en la transmisión del docente-, un abordaje de los saberes en torno a la resolución de problemas que requieren diferentes enfoques disciplinares, la incorporación de nuevas tecnologías con la inclusión del uso de los dispositivos informáticos bajo el modelo 1 a 1 , entre otras innovaciones que se encuentran a tono con las nuevas pedagogías.

Encontramos en las propuestas curriculares diferentes maneras de legitimar su elaboración. Las escuelas privadas católicas 1 y 2 presentan su oferta académica en consonancia con las políticas y orientaciones curriculares nacionales que luego combinan con su especificidad de escuelas católicas. A fines del siglo XX, la escuela católica 1 encaró diversas iniciativas (como olimpíadas de historia y matemáticas, torneos de rugby, actividades de acción social, coro, orquesta de cámara, entre otras) con el objeto de adaptar su oferta tradicional a los cambios socioculturales de la época (Información/ web institucional, escuela católica 1).

\footnotetext{
${ }^{5}$ Hemos podido identificar la existencia de proyectos pedagógicos entre disciplinas en algunas de las escuelas, pero su presencia es más bien lateral y de carácter eventual.
} 
El análisis de la presentación institucional indica que estas escuelas se posicionan frente a un campo de regulaciones y se interpretan a sí mismas como entes con iniciativas que generan una propuesta particular. La diversidad de actividades de las escuelas católicas no es el mero resultado de una suma. Las escuelas "eligen" determinados bienes-propuestas que consideran valiosos para las familias, para ellas como instituciones y para la apuesta que ambos realizan para sus hijos/as. Ese conjunto de iniciativas muestra a estas escuelas ofreciendo productos o experiencias culturales asociables a una "alta cultura" (orquestas, por ejemplo), junto a otras más habituales dentro del sistema educativo (olimpíadas) o más particulares en torno a los sectores que interpela (el rugby como práctica de sectores medios altos y altos).

En la propuesta curricular del colegio católico 1, por ejemplo, hallamos una visión escolástica, tomista, de la persona a ser "educada": supone un "todo integrado", un sujeto que se armoniza mediante la formación a partir de una pedagogía espiritualista (Información/ web institucional, escuela católica 1). Al mismo tiempo, las escuelas católicas 1 y 3 mencionan el imperativo de incorporar las nuevas tecnologías de la educación, pero las subsumen a otras finalidades trascendentes. El conjunto de las cinco escuelas plantea la necesidad de contar con saberes actualizados para continuar en la universidad, a la que los egresados de estas instituciones se supone que "naturalmente acceden" o deben hacerlo. Son hijos y, en muchos casos, nietos de universitarios.

El colegio católico 3 también resignifica el plan de estudios oficial y su tradición irlandesa, en una combinación entre exigencia académica y atención a las habilidades y capacidades individuales. Desarrolla, por un lado, una pedagogía personalizada basada en el "desarrollo y crecimiento según las potencialidades" de sus alumnos; y por otro lado, plantea una resignificación psi del currículum, atento, por ejemplo, a prácticas de inclusión de jóvenes con discapacidades de distinto tipo (motriz, cognitiva) o con "déficit de atención". Aquí la inclusión de/en la diversidad funciona como paradigma pedagógico que requiere adecuaciones curriculares específicas-con la intervención de psicopedagogos-que permiten construir "una escuela que incluye a todos", como relataba una docente. La escuela señala en su presentación institucional: "También aspiramos a que cada alumno sea un líder proactivo, dueño de su propio destino. Esto le permitirá pensar y modificar su propia realidad y la de su comunidad, con nuestro sello distintivo".

Si bien todas las escuelas que forman parte de la muestra son instituciones que tienen por lo menos 50 años de antigüedad, resignifican su pasado y su identidad institucional (lo irlandés, lo católico, etc.) de modos distintos: adaptándose a los cambios (lo tecnológico en la católica 1, las nuevas "diversidades" en la católica 3) o asumiendo un lenguaje que proviene del management, que no por eso olvida la identidad católica o institucional en la que aspira formar. 
¿Cómo hacemos, cada año, para responder al objetivo de fortalecer la formación de sujetos de derecho, con capacidad de ejercer y construir ciudadanía? Éstos son nuestros proyectos:

—Modelo de Poder Legislativo.

-El voto a los $16 \ldots$

—Construcción de Ciudadanía...

—Taller ONU... (Información web institucional, escuela católica 3).

El estilo de formación institucional se construye generando una propuesta de enseñanza que articula prácticas curriculares y áulicas con instancias de formación extra-áulicas, como puede ser un Taller ONU, donde estudiantes de la misma escuela y luego entre escuelas debatan temáticas vinculadas a las relaciones internacionales. Estas actividades abonan la tesis de que se trata de apuestas por formar ciudadanos comprometidos que se familiaricen con los mecanismos institucionales del poder (Congreso de la Nación, ONU, voto, etc.).

Por otra parte, la escuela pública universitaria y la privada laica presentan su propuesta curricular independientemente de las fuentes locales que nutren la elaboración de la misma. En el primer caso es la autonomía de las universidades la que explica el desarrollo de programas propios fundados en el conocimiento de los profesores universitarios. La presentación institucional refiere a la estructura departamental y a cada uno de los programas de estudio sin mencionar otras especificaciones. En consonancia con su pertenencia universitaria, esos programas presentan una estructura a imagen y semejanza de los programas de estudios de nivel superior.

El colegio privado laico, por su parte, se autoproclama como una escuela "vibrante, interesada por el cuidado del otro que presenta elevados logros como institución bilingüe". El plan de estudios está legitimado en la presentación institucional por las directrices del programa del Bachillerato Internacional que vertebra la organización curricular y las prácticas de enseñanza. La perspectiva de la escuela apela al desarrollo individual centrado en formar las competencias para las sociedades del siglo XXI. Estas características se combinan con la mención de sus principios fundacionales, su referencia al origen presbiteriano de la institución, su actual apertura religiosa y la formulación de la "persona" como "ser intelectual, emocional, físico y espiritual". Estos principios se articulan con una presentación basada en la innovación y la iniciativa de sus estudiantes, es decir, combina la presentación de su acervo y tradición institucional —que la vincula con su pasado- con la referencia a las potencialidades de los estudiante para que "experimenten la emoción de descubrir que son capaces de lograr más de lo que creían posible y los alentamos a abrazar nuevas 
oportunidades y desafíos, y apoyarlos en el desarrollo de la resiliencia y la determinación" (Información/web institucional, escuela privada laica).

El conjunto de las escuelas está consustanciado con la formación mediante un currículum generalista orientado a la consecución de estudios de nivel superior, con variantes según se trate de una propuesta centrada en procesos de enseñanza más clásicos o con cambios orientados a lo que las escuelas interpretan como los requerimientos del "nuevo siglo". Los principios de accountability, efectividad y los indicadores mensurables no parecen tener un lugar de relevancia en el modo en que organizan su propuesta curricular, aunque ese discurso permea en algunas presentaciones institucionales.

\section{Espacio, infraestructura escolar y equipamiento: una estética de privilegio}

En las consideraciones que las familias realizan sobre las escuelas, el edificio, la infraestructura y el lugar en la ciudad donde se encuentran son dimensiones destacadas y a veces nombradas como principal motivo de la elección escolar. La valoración delos espacios se construye al mismo tiempo que se eligen lugares similares para vivir. Como dicen Gaztambide-Fernández y Howard (2010) y Kahn (2011), los espacios escolares son personajes importantes en las historias de escolaridad de las elites. La exclusión se instala en los campus a través de la arquitectura y los jardines y terrenos que rodean el edificio escolar, creando un espacio diferencial. Sus fronteras espaciales y arquitectónicas buscan brindar una experiencia donde la estadía sea agradable y cómoda en función de determinadas sensaciones de bienestar y belleza.

En la presentación de las escuelas católicas 1 y 2 se conjugan la dotación de infraestructura adecuada con un desplazamiento hacia la zona norte que va de la mano con el desplazamiento residencial de los sectores a los que el colegio atendía.

(Nombre de la escuela) comienza sus actividades en plena Capital Federal. Era necesario hallar un predio que, por su extensión, hiciera posible dotarlo de un campo de deportes y de jardines, a fin de crear una atmósfera de tranquilidad alrededor de las aulas. Luego de una intensa búsqueda, se encontró el lugar ideal para construir el soñado colegio, y su campo de deportes, en el barrio de La Horqueta, San Isidro (Información web institucional, escuela católica 1).

Esa idea acerca de los perjuicios de la vida urbana y los beneficios de la suburbana configuraron un paisaje de ciudad y de "lugar" producido para una formación "tranquila" y armónica de los hijos de estos sectores. En los casos relevados, las escuelas gozan de una historia hecha tradición. Como indicamos, las instituciones tienen por lo general más de 50 años de existencia, ${ }^{6}$ y eso permite ver en sus edificios la respuesta

\footnotetext{
${ }^{6}$ Es ello lo que posibilita que exalumnos envíen a sus hijos y nietos a la misma escuela, construyéndose así la elección escolar en una tradición familiar.
} 
institucional a las transformaciones urbanas. La escuela católica 1 decide su traslado al conurbano norte, con los riesgos de modificar su alumnado. Sin embargo, el desplazamiento institucional del centro de la Ciudad de Buenos Aires no resultaba novedoso para su matrícula, aun cuando es considerable la distancia que recorren diariamente muchas de estas familias para llegar a la escuela. Es que la suburbanización de las familias de grandes ingresos fue un proceso gradual. Muchas de ellas todavía hoy viven en el centro de la Ciudad de Buenos Aires y se trasladan hacia la zona norte de la Provincia de Buenos Aires para concurrir a la escuela. A pesar de la distancia y la inversión de tiempo que eso requiere, dos elementos acompañan la continuidad de esa práctica: la apuesta familiar por estas escuelas y la configuración de un circuito urbano (Magnani, 2002) que les resulta "familiar", en el sentido nativo del término, ya que familiares y amigos circulan y residen allí (Fuentes, 2015a). La estética producida en ese desplazamiento responde a procesos de significación cultural que si bien hoy se agrupan bajo el country life (Ballent, 2014), tienen una historia centenaria de producción de sentidos sobre la naturaleza y la "civilización" del paisaje. En esa estética, el espacio habitado y el educativo-formativo son cruciales para producir la experiencia de contacto con la "naturaleza" y sus beneficios formativos, y una sensación de privilegio, en el sentido de que se habita un extenso y costoso espacio al que pocos acceden.

En la relación con el espacio que propician notamos una estrategia de adaptación de las escuelas: ya no alcanza con presentar su historia y su tradición. El pasado es combinado con una preocupación por el presente, la renovación y la actualización, junto a una dotación de equipamiento e infraestructura asociados a un capital diferencial en relación con otras escuelas. Esa producción diferencial combina infraestructura con concepciones pedagógicas. Identificamos una inquietud por mejorar las condiciones edilicias: laboratorios, teatro, "multicanchas de volley, handball y básquet y la sala de pesas y ejercicio; (...) las obras de nivelación, sembrado, canalización y riego automático computarizado en todo el campo deportivo; el playón de estacionamiento", son parte del equipamiento destacado por la escuela católica 1 (Información web institucional, escuela católica 1). Nótese la combinación del peso otorgado a la infraestructura deportiva -instancia de formación física y "moral" de estos jóvenes - con la connotación "tecnológica" con que la escuela se presenta a sí misma, combinando historia y tradición con una infraestructura que responde a las expectativas que depositan en las prácticas deportivas, por un lado, y la orientación en la formación para el futuro, planteándose como una institución en continua actualización. Por esa razón las escuelas se presentan permanentemente en "obra", "creciendo", y al mismo tiempo "adaptándose a los cambios".

La escuela católica 3 se instaló originariamente en una localidad del conurbano que tempranamente en el siglo $\mathrm{XX}$ fue un lugar de residencia de familias inglesas, irlandesas y francesas; y fue constituyéndose como un lugar "verde", de descanso y recreación deportiva _-por el club vecino al río Reconquista, donde se practicaban 
deportes náuticos-. La infraestructura y el equipamiento son fundamentales al momento de su presentación institucional, pero además se construye en la experiencia de "estar" en la escuela:

Mientras estoy en una galería del primer piso conversando con una profesora, veo a una de las directoras de la escuela que acompaña a dos adultos, un varón y una mujer, mostrándoles la infraestructura. Pasa por el laboratorio de ciencias naturales, luego recorren la galería e ingresan a unas amplias aulas. Salen de allí y les muestra el patio del nivel primario que se ve hacia abajo. Más allá, una parte del parque del colegio: señalan ambos un grupo de estudiantes que están sentados allí en círculos junto a una profesora. Los miro y algunos están medio acostados, otros inclinados, algunos tomando nota, parecen estar conversando de algún tema que, a pesar de lo relajado de sus posiciones, sostiene algo de la atención del grupo. Luego, la directora y la pareja siguen caminando por la gran galería e ingresan al laboratorio de computación (Diario de campo, Sebastián Fuentes, 2009).

La incorporación de nuevos grupos familiares se produce, entre otros atributos, por medio del atractivo o las ventajas y diferencias que este tipo de escuelas presentan. Es el tipo de experiencia que suscitan, entre la tecnología y la naturaleza, entre el abundante equipamiento y los históricos edificios, entre un pasado "del que estar orgulloso" y un presente en constante innovación. Al menos en el caso de la escuela católica 3, se fomenta una experiencia relajada, que evoca un romanticismo (Ballent, 2014) en el que la figura de los jóvenes leyendo un texto bajo un árbol del hermoso parque constituye una de las imágenes de una pedagogía del privilegio. Esa excepcionalidad o unicidad de la experiencia es retomada por los egresados en sus trayectorias, reconstruyendo una experiencia de aprendizaje asociada al bienestar y disfrute:

Yo amé poder estar afuera y es otro tipo de contacto. Tras que estás todo el día en el colegio por lo menos sentía el sol, el pasto, el perfume de las flores, no sé, corrés afuera, hay perfume, ¿viste? Yo me acuerdo mucho las sensaciones no solo académicas sino de estar, bueno, en un lugar lindo, que realmente te descansa mucho más que estar en un patio. Yo me negué rotundamente a poner a mis hijos en un colegio acá en el centro por eso exclusivamente. Hay colegios excelentes pero yo quería que ellos vuelvan quemaditos, cansados de correr, de jugar afuera (egresada de la escuela católica 2, comunicación personal).

La importancia manifiesta dada "al verde" o la poca relevancia del trayecto a recorrer todos los días para llegar a la escuela expresan, como señala Bourdieu (1984), un desplazamiento del interés desde "el contenido" hacia "la forma". O, reformulando las afirmaciones del sociólogo francés, uno de los modos de construir diferencias es "comprometer los principios de una estética 'pura' en las opciones más ordinarias de la existencia ordinaria" (p.37). 


\section{Convivencia y "disciplina": preocupación constante, dispositivos diferenciales}

Enseñar el comportamiento adecuado constituye una preocupación de estas escuelas, aunque claro está no es exclusivo de ellas. El análisis de los dispositivos que se desarrollan institucionalmente para regularla da indicios sobre el tipo de relaciones que buscan construir entre estudiantes y entre estudiantes y docentes. En el análisis realizado sobresalen las propuestas de las escuelas pública universitaria, católica 3 y privada laica.

En la escuela católica 3, el rol de los preceptores es realzado: "controlar que los pendejos no hagan cagadas", es lo primero que relata un preceptor. Pero a continuación aparecen nuevos elementos: "te ponés a conversar con ellos en los recreos, o cuando los mandan a preceptoría también, te cuentan cosas de su vida, lo que les pasa, los problemas que tienen" (Diario de campo, Sebastián Fuentes, 2009). La tutoría adulta se ejerce no solo en torno a la conducta en la escuela sino también sobre la vida de los jóvenes afuera de la institución. Ello constituye la tarea de preceptores y docentes "tutores" que deben saber las problemáticas de los estudiantes. Saber "lo que les pasa" contribuye a formar un dispositivo de personalización que construyen los preceptores con los estudiantes y que permite sostener una relación más cercana que la mantenida con los docentes.

Como todas las escuelas, ${ }^{7}$ la católica 3 tiene un acuerdo institucional de convivencia. Todas las escuelas de la provincia deberían producir su propio acuerdo con la participación activa de toda la comunidad educativa. En esta institución, además, se le da un tinte particular: desde fines de los 90 desarrollan un programa de convivencia y mediación escolar, actividad relevante cuando se analiza la formación de un sentido de "liderazgo" desde una perspectiva "convivencial". El programa es llevado adelante por una psicopedagoga, y originalmente desarrollado por un abogado especialista en mediación. En él se canalizan conflictos de convivencia entre estudiantes y, en ocasiones, situaciones entre docentes y alumnos. Allí se tratan y en caso de diferendos se busca conciliar a las partes en conflicto. También interviene en situaciones de conflictos grupales, ante casos de hostigamiento a estudiantes. De este modo, una política provincial es reapropiada por la institución en un programa que le ofrece la oportunidad de diferenciarse.

Los estudiantes que forman parte del consejo realizan un curso anual de una semana intensiva de duración, destinado a formar "alumnos mediadores". Ese dispositivo jerarquiza a los alumnos/as que lo realizan, en el sentido de que son vistos por sus compañeros como "conocedores" de "este tema" y suelen ser elegidos para

7 De acuerdo con las políticas desarrolladas en la provincia de Buenos Aires, los Acuerdos Institucionales de Convivencia (AIC) constituyen herramientas que todas las instituciones deben desarrollar con la participación de los actores institucionales. 
participar del consejo. En el curso se presentan situaciones, análisis de casos, donde se resalta el rol social del mediador, en un nuevo énfasis en la formación hacia la "responsabilización" de sí mismo por los problemas y conflictos sociales.

De un modo similar, la escuela privada laica desarrolla un sistema de convivencia con representantes estudiantiles en un órgano que comparten con las autoridades del establecimiento. Ese consejo es visualizado como vital para el aprendizaje de habilidades sociales de los estudiantes, que son destacados por su condición de líderes, resultan mencionados con nombre y apellido en la página web de la institución, y tienen una vestimenta que los diferencia de sus compañeros. La escuela presenta una trama de instancias que contribuyen a fomentar la disciplina (tutores, coordinadores de curso, consejo de autoridades y estudiantes, preceptores, etc.). En general, estas escuelas desarrollan una política que explícitamente aborda la potencialidad del conflicto convivencial, combinando un tipo de experiencia que enfatiza el "estar bien" y un liderazgo para la resolución de los problemas.

El colegio de gestión universitaria cuenta con un consejo en donde los alumnos tienen representación estudiantil, junto con los profesores y las autoridades. A diferencia de los casos anteriores, los alumnos se organizan en consonancia con pertenencias políticas que tienen su correlato con agrupaciones de partidos políticos o movimientos estudiantiles universitarios. El aprendizaje de la militancia política es un elemento nodal e histórico de esta institución y canaliza, además, la sociabilidad y los contactos que son centrales en el proceso de formación que busca producir esta escuela.

\section{Viajar "afuera" y "adentro": estudiantes globales, ciudadanos locales}

Los viajes constituyen una experiencia cotidiana para los alumnos de estas escuelas: en algunas de las instituciones privadas los/ las docentes suelen referirse a las adaptaciones que la escuela hace de la normativa escolar debido a las inasistencias de los alumnos, que se ausentan en numerosas ocasiones por los viajes que hacen con sus familias. Además de la experiencia familiar de viajes internacionales, los tres colegios católicos ofrecen propuestas en el exterior que involucran a algunos alumnos en función de actividades que los jóvenes realizan en la escuela. A modo de ejemplo, los alumnos de la escuela católica 1 viajan a Estados Unidos y a Perú. En los blogs que los estudiantes realizan relatan la experiencia de ir a tomar cursos de anatomía y cálculo en una escuela estadounidense. Destacan con naturalidad las actividades allí realizadas y con sorpresa cuestiones como "tener que dormir en el piso" en ocasión de visitar los dorms de alumnos de esas universidades, pero expresan sin más asombro que en la visita al campus: "nos dieron nuestros iPads", "Gonzalo comparó Buenos Aires con New York", "presentamos Argentina: ubicación geográfica, alimentos, clima, costumbres como el mate, etc." (Información/ blog de estudiantes y web institucional, escuela católica 1). Junto a la experiencia familiar de circulación internacional con 
finalidad turística o recreativa, estos jóvenes van adquiriendo un posicionamiento global, en el modo de circulación, en la comparación que allí construyen (Buenos AiresNueva York) y en ellos mismos como articuladores globales de esos lazos, que en el caso descrito además los pone como representantes de la nación.

En la escuela católica 3 los estudiantes más avanzados en su nivel de inglés realizan un viaje a los Estados Unidos. En ese país los reciben colegios secundarios que pertenecen a la misma congregación, siendo instituciones reconocidas a nivel local. Permanecen durante un mes residiendo en la casa de algunos de los jóvenes que luego, al año siguiente, vendrán a Buenos Aires. En los dos casos, los estudiantes realizan la misma vida cotidiana escolar que los estudiantes que los reciben. Cuando llegan a la escuela, los estudiantes extranjeros participan de las actividades curriculares y extracurriculares. "Cuando vinieron las yanquis, se re sorprendían de las cosas que hacemos en el barrio. Ellas tienen allá algo así de community service, nosotras fuimos, pero es como que la realidad no es tan cruda como la de acá", relataba una de las jóvenes entrevistadas. En ese circuito de viajes, la participación en actividades "solidarias" o comunitarias hace a la circulación de determinados saberes sobre la marginalidad y la pobreza. Un currículum solidario global se extiende entre esas escuelas. Lo que tienen en común es que la circulación de esos saberes produce un posicionamiento sobre/en la desigualdad construida a nivel internacional. En general, se trata de la opción por un circuito estadounidense asociado al bilingüismo de estas escuelas.

En la escuela universitaria, dada la referencia europea, los viajes se realizan a Paris y Roma. Los estudiantes deben postularse y son seleccionados según su conducta y calificaciones. En el transcurso del intercambio asisten a escuelas de características semejantes a la propia, desarrollan visitas de carácter cultural y a su regreso deben presentar una monografía que es evaluada.

Los viajes internos también son frecuentes, constituyen el destino de dos dispositivos curriculares de estas escuelas: los viajes recreativos o de estudios, asociados al conocimiento del lugar y de la experiencia de "vida en la naturaleza". Entre el conocimiento escolar, la convivencia y la recreación, y el contacto con la naturaleza y el paisaje "nacional", en ocasiones este tipo de viaje se combina con otro dispositivo: los viajes de estilo "solidario", ya sea religioso, al modo de la misión católica, o de servicio a alguna comunidad marginada o pobre de la Argentina. Se recrea en ellos un centrismo: el encanto por el "interior" del país, en una visión del mismo que contribuye a pensarse en el centro, y exotizar y romantizar la periferia. En este tipo de propuestas se complementa una experiencia de estudiante global con la del ciudadano local/nacional. Así como se aprende y acostumbra el viajar —entre la familia y la escuela - se naturaliza la posición de centralidad dada históricamente a la capital porteña que estos jóvenes aprenden en estas instancias de circulación. Viajar, recrear y ayudar forman parte de los saberes a los que apuestan y que los diferencian por los circuitos de pertenencia. 


\section{El énfasis en el currículum "solidario" (o aprender la jerarquía)}

Madres y docentes, en primer lugar y, padres en segunda instancia (en una división social del trabajo por género) trabajan en conjunto -aunque no necesariamente de manera armónica- en la formación del carácter moral de los jóvenes. En las escuelas católicas 1 y 2 los sujetos ejercitan una modalidad de ayuda ligada a los valores religiosos y a la caridad. Espacios como tés solidarios, cenas, partidos de jockey entre madres, partidos de golf, etc., son experiencias formativas que trascienden lo que ocurre ese día y durante el tiempo que dura el evento.

En la escuela privada laica se producen las mismas actividades, pero sin el contenido religioso. Las actividades de recaudación de fondos para eventos, las invitaciones y la publicidad de los mismos, las decisiones en torno a la organización, los días de ensayo y pruebas, las revistas o los catálogos que realizan, los avisos que allí aparecen son parte del aprendizaje e involucran a distintos sujetos que entran en contacto no solo el día del suceso ni únicamente en los espacios en donde trascurre el "encuentro solidario". Los alumnos preparan estos acontecimientos con anticipación. Las donaciones de alimentos que se servirán durante una comida surgen de contactos y de los recursos a movilizar por las familias de la escuela. Se sortean objetos realizados por las mismas madres o los alumnos en sus actividades extraescolares.

Los temas convocantes de cada reunión colaboran con reforzar los valores de cada institución. Estos espacios resultan privilegiados para la socialización y la construcción de redes entre los alumnos y, a través de ellos, para sus familias. Las actividades solidarias, como ámbito de encuentro e intercambio para el grupo doméstico, especialmente para las madres, son parte integral del currículum y son valoradas.

Existen materias como "Apertura a la comunidad". La idea de una materia así surge para sacar a los alumnos "de la realidad en la que viven que es solamente su realidad, sus vacaciones en los mismos lugares con la misma gente, ahora los barrios privados, universidad privada y bueno, entonces la idea es que un poco conozcan que existen otras cosas" (Norma, profesora escuela católica 2). Esta iniciativa no es una propuesta aislada. Varias instituciones tienen "acción social como materia en secundaria". Aunque varíen sus nombres, las escuelas desarrollan estas prácticas, ya sea por materias o por proyectos interdisciplinarios, que tienen una "salida" a la comunidad. Algunas instituciones incentivan también la participación de los docentes en proyectos mediante la metodología del aprendizaje-servicio (Fuentes, 2015a). Además, en las escuelas católicas que desarrollan actividades "pastorales"8 de manera extracurricular, se realizan actividades como visitas a los hospitales públicos, o apoyo escolar en centros comunitarios o capillas de algún barrio carenciado cercano. Entre

${ }^{8}$ Se trata de actividades que podrían denominarse "solidarias", pero que conjugan por lo general una motivación religiosa y pueden estar acompañadas de prácticas explícitamente evangelizadoras. En la organización escolar suelen existir áreas o departamentos de "pastoral". 
sus actividades solidarias las escuelas católicas también realizan "misiones". Si bien ello guarda un sentido evangelizador y de conversión, el significado de esa actividad a veces reduce la tarea en relación con la evangelización y la traduce o actualiza en torno a una "presencia" entre los pobres, al énfasis en el "compartir" con los que "menos tienen".

Entrevistador: ¿Qué hacían en la misión?

Entrevistado: Visitar casas, a veces llevábamos la Biblia, leíamos una lectura (bíblica), pero si no, no era importante, o sea, era ir y compartir, estar con la gente, qué se yo (...) darte cuenta de que en realidad a pesar de que no tienen nada, tienen más que vos, (...) vas a dar y recibís un montón (Egresado escuela católica 3, 21 años, comunicación personal).

Estas ofertas de las escuelas se inscriben en la historia de la caridad que tradicionalmente han desarrollado los sectores católicos. Las acciones solidarias asumen una preocupación por lo que las perspectivas más conservadoras han llamado "diversidad": se ayuda a los pobres, a los ancianos, etc. Como señalan Tiramonti y Ziegler (2008), la participación solidaria es voluntaria y toma formas aparentemente apolíticas a través de las cuales se reemplazan demandas basadas en derechos por carencias. La buena voluntad detrás de estas opciones transforma derechos en asistencialismo: los otros devienen "necesitados", receptores de regalos y donaciones (pp.126 y 128). Existe en muchos casos una construcción sobre la desigualdad social que se produce en estas instancias, solo que se trata de una desigualdad que la relación de ayuda resuelve en un vínculo interpersonal (Saint Martin, 1993).La escuela enseña un modo de "dar" tiempo, el sentimiento de haber cumplido con un deber, de haber hecho fructificar los talentos de los que los estudiantes son depositarios (Saint Martin, 1993). Forma en una idea de responsabilidad: hacerse cargo de una sociedad que los necesita y sobre la cual ellos pueden y deben actuar (Fuentes, 2015a).

A través del aprendizaje solidario los sectores privilegiados refuerzan su estatus de elite y al calor de la extensión de la ideología de la "responsabilidad social" de las empresas, de los empresarios, de las fundaciones, la responsabilización por la pobreza pasa a ser un nuevo capital, que remarca la trayectoria y la experiencia de estos jóvenes.

\section{CONCLUSIONES. EL CURRÍCULUM COMO APUESTA A LA DIFERENCIACIÓN}

La internacionalización, los viajes y el contacto con la pobreza en sus distintas variantes producen relaciones con los saberes, es decir, implican un modo de concebir el currículum como una propuesta que supone que en esos dispositivos se aprende a ser un ciudadano local comprometido, "solidario", a conocer el mundo y manejarse en él, circular. Ello, al vincularse con dimensiones cotidianas, como una infraestructura- 
estética y un "estar siempre en obra", "creciendo", constituyen dimensiones más sutiles, pero no menos relevantes en la formación en los valores e ideologías a los que apuestan las familias y las escuelas. No se trata solo de elegir una escuela cuya infraestructura es claramente distinta, más amplia, costosa incluso, o "más bella" que las restantes; sino también de un vínculo entre el estilo de vida que los distingue 0 al que aspiran y un modo de entender la relación con el espacio que produce la experiencia y los recuerdos de una educación tan distinguida como selecta. A su vez, transformar los conflictos de convivencia en una nueva instancia para formar "líderes" que resuelvan las problemáticas interpersonales, o apostar a la formación de la camaradería por medio de houses o de la militancia política, son estrategias formativas en un currículum que no cesa de sumar todos sus esfuerzos para una educación de y en el privilegio y el liderazgo.

Las dimensiones que hemos analizado de las escuelas que se posicionan y apuestan a la educación de los sectores de elite nos indican que el currículum es una pieza clave de la diferenciación.

Las instituciones orientadas a las elites desarrollan un currículum complementario al oficial que rige a las escuelas del conjunto del país, elementos distintos para la formación de estos grupos, creando un currículum "a medida y exclusivo"' (Ziegler, Gessaghi y Fuentes, 2018). Ahora bien, las dimensiones que analizamos en este trabajo nos indican que ese currículum complementario es una condición necesaria pero no suficiente en la conformación de las propuestas de enseñanza de las escuelas de elite. No se trata de una simple suma de actividades, disciplinas y dispositivos — que está presente - como si el capital económico de las familias se trasladara a las escuelas y ellas pudieran ofrecer todo lo que no ofrecen las escuelas estatales o las privadas de menores ingresos. Sino también de una serie de experiencias de aprendizaje, de presentación pública y de relación con los saberes que implican una apuesta por una formación que familiarice a los estudiantes con posiciones, estéticas y "valores" distintivos. Se trata de un currículum que en cuanto selección es producido por las mismas escuelas que seleccionan saberes y experiencias, reorientando las políticas educativas -el currículum prescripto- en función de un "paquete" que no es ni tan homogéneo — no todas escuelas ofrecen lo mismo - ni tan heterogéneo — como hemos visto, algunas propuestas como el tipo de viajes son comunes - lo cual les permite profundizar una diferencia en dimensiones sutiles y explícitas de la experiencia escolar.

Al construir sus propuestas escolares se ubican entre lo nuevo y lo viejo, lo nacional y lo global, aunque en la organización del plan de estudios el énfasis recaiga en el currículum "clásico". La propuesta curricular solo en algunos casos hace gala de

${ }^{9}$ Esta no es una práctica exclusiva de estas escuelas, dado que las instituciones que no pertenecen al segmento de las elites también agregan al currículum oficial otras actividades, como lo indicamos en publicaciones previas (Ziegler, 2014; Gessaghi, 2014; Fuentes, 2015b), por eso el acento puesto en el tipo de actividades que proponen las primeras. 
su innovación, y cuando lo hace (escuela privada laica) la propuesta pedagógica no deja de apelar a un pasado, que también se recrea en la estética del privilegio: modernizar lo antiguo y fundacional, actualizar el prestigio.

Situado en el campo de investigaciones sobre la desigualdad socioeducativa y las discusiones acerca de la educación secundaria en la Argentina y la región, este trabajo comenzó por preguntarse por la relación entre un plan de estudios común prescripto en el sistema educativo argentino y los modos de diferenciación que operan en escuelas que atienden a estos sectores sociales. Sin embargo, es necesario seguir indagando tanto sobre los modos de (re)producir el privilegio en la vida cotidiana escolar como sobre las apropiaciones y negociaciones de las políticas educativas "comunes" que realizan las instituciones. Profundizar esta área de vacancia es indispensable para documentar cómo en contextos de ampliación del derecho a la educación el sistema educativo, en su funcionamiento cotidiano, proporciona insumos diferenciados a los distintos grupos sociales.

\section{REFERENCIAS}

Albornoz, F.,Furman, M., Podestá, M. E., Razquin, P., y Warnes, P. E.(2016).Diferencias entre escuelas privadas y públicas. Revista Desarrollo Económico, 56(218), 3-31.

Ballent, A. (2014). Country life. Los nuevos paraísos, su historia y sus profetas. En A. Ballent y J. Liernur (Comps.), La casa y la multitud. Vivienda, política y cultura en la Argentina moderna (pp.627-53). Buenos Aires, Argentina: Fondo de Cultura Económica.

Bernstein, B. (1988). Clases, código y control. Madrid, España: Akal.

Bourdieu, P. (1984). La distinción. Criterio y bases del gusto. Barcelona, España: Taurus.

Braslavsky, C. (1985). La discriminación educativa. Buenos Aires, Argentina: FLACSO/ Miño y Dávila.

Canelo, P. (2012). ¿Sabios, ricos y buenos? Perfiles sociales y carreras políticas de los senadores nacionales argentinos en 1973, 1983 y 1989. En S. Ziegler y V. Gessaghi (Comps.), Formación de las elites. Investigaciones y debates en Argentina, Brasil y Francia (pp. 203-225). Buenos Aires, Argentina: Manantial.

Cerletti, L. (2014). Familias y escuelas. Tramas de una relación compleja. Buenos Aires, Argentina: Biblos.

Cervini, R. (2003). Diferencias de resultados cognitivos y no cognitivos entre estudiantes de escuelas públicas y privadas en la educación secundaria de Argentina: un análisis multinivel. EducationPolicyAnalysis Archives, 11(5), 1-32.

Dallaglio, L. (septiembre de 2015). Distinción y prácticas sociales en una universidad privada de elite del Área Metropolitana de Buenos Aires. Presentado en V Jornadas sobre Etnografía y Procesos Educativos, IDES. Buenos Aires, Argentina.

De Imaz, J. L. (1964). Los que mandan. Buenos Aires, Argentina: Eudeba. 
Dussel, I. (1997). Currículum, humanismo y democracia en la enseñanza media (1863-1920). Buenos Aires, Argentina: FLACSO/Universidad de Buenos Aires.

Fabrizio, M. L., y Montero, J. (julio de 2014). Prácticas presentes y nociones de futuro: consideraciones adultas en torno a las trayectorias de niños y jóvenes en contextos de desigualdad social. Ponencia presentada en el XI Congreso Argentino de Antropología Social, Universidad Nacional de Rosario. Rosario, Argentina.

Fuentes, S. (2015a). Educación y sociabilidad en las elites de Buenos Aires (Tesis de doctorado). Instuto de Altos Estudios Sociales, Universidad Nacional de San Martín, San Martín, Argentina.

Fuentes, S. (2015b). La formación moral de los jóvenes de elite en circuitos de educación privada en Buenos Aires. Pro-Posições, 26(2), 75-98. https://dx.doi.org/10.1590/0103-7307201507704

Ganimian, A. J. (2013). No logramos mejorar: Informe sobre el desempeño de Argentina en el Programa para la Evaluación Internacional de Alumnos (PISA) 2012. Recuperado de http://educar2050.org.ar/wp/wp-content/uploads/2015/08/Informe-PISA-Argentina-20121.pdf

Gaztambide-Fernández, R., y Howard, A. (2010). Conclusion: Outlining a Research Agenda on Elite Education. En Educating Elites: Class Privilege and Educational Advantage (pp.195-209). Lanham, MD: Rowman \& Littlefield Education.

Gessaghi, V. (2013). Familias y escuelas: sentidos acerca de la educación en la clase alta argentina. Revista RUNA, 34(1), 73-90.

Gessaghi, V. (2014). Elogio de los imprevistos. Una experiencia etnográfica con "la clase alta". Revista Ensambles, 1(1), 157-171.

Gessaghi, V. (2016). La educación de la clase alta argentina. Entre la sangre y el mérito. Buenos Aires, Argentina: Siglo XXI.

Goenaga, M. (2016). "Bien de familia". Capitales, sucesión y trayectorias formativas en una familia empresaria (Tesis de grado). Universidad de Buenos Aires, Buenos Aires, Argentina.

Heredia, M. (2012). ¿La formación de quién? Reflexiones de la teoría de Bourdieu y el estudio de las elites en la Argentina actual. En S. Ziegler y V. Gessaghi (Comps.), Formación de las elites. Investigaciones y debates en Argentina, Brasil y Francia (pp. 277-295). Buenos Aires, Argentina: Manantial.

Kahn, S. R. (2011). Privilege. The making of an adolescent elite at St. Paul's School. New Jersey, NJ: Princeton University Press.

Kessler, G. (2002). La experiencia escolar fragmentada. Estudiantes y docentes en la escuela media de Buenos Aires. Buenos Aires, Argentina: IIPE-UNESCO.

Landau, M. (2011). Política y participación ciudadana en la Ciudad Autónoma de Buenos Aires. Buenos Aires, Argentina: Miño y Dávila.

Luci, F. (2012). La educación de los dirigentes de empresas: la formación en negocios y el acceso a la cúpula de las principales organizaciones. En S. Ziegler y V. Gessaghi (Comps.), Formación de las elites. Investigaciones y debates en Argentina, Brasil y Francia (pp. 227-247). Buenos Aires, Argentina: Manantial. 
Magnani, J. G. (2002). De perto e de dentro: notas para uma etnografía urbana. Revista Brasileira de CiênciasSociais, 17(49),11-29. https://doi.org/10.1590/S0102-69092002000200002

Méndez, A. (2013). El colegio. La formación de una elite meritocrática en el Nacional Buenos Aires. Buenos Aires, Argentina: Sudamericana.

Narodowsky, M., y Moschetti, M. (2015). The growth of private education in Argentina: evidence and explanations. Compare, 45(1), 47-69.

Puigróss, A. (1990). Sujetos, disciplina y currículum en los orígenes del sistema educativo. Buenos Aires, Argentina: Galerna.

Rivas, A. (2010). Radiografía de la educación argentina. Buenos Aires, Argentina: CIPPEC.

Rodríguez Moyano, I. (2012). Capital cultural y estrategias educativas de las clases altas de la Ciudad de Buenos Aires. En S. Ziegler y V. Gessaghi (Comps.), Formación de las elites. Investigaciones y debates en Argentina, Brasil y Francia (pp. 147-163). Buenos Aires, Argentina: Manantial.

Sacerdote, B. (2011). Peer effectsin education: How might they work, how big are they and how much do we know thus far?. Handbook of economics of education, 3, 249-277.

Saint Martin, M. (1993). L'espace de la noblesse. París, Francia: Éditions Métailié.

Santillán, L. (2012). Quienes educan a los chicos. Buenos Aires, Argentina: Biblos.

Sarrabayrouse Olivera, M. J. (1999). Grupos, lealtades y prácticas: el caso de la justicia penal argentina. Revista de Sociologia e Política, 13, 81-104.

Tedesco, J. C. (1986). Educación y sociedad en la Argentina 1880-1945. Buenos Aires, Argentina: Ediciones Solar.

Tiramonti, G. (2004). La fragmentación educativa y los cambios en los factores de estratificación. En G. Tiramonti (Comp.), La trama de la desigualdad educativa. Mutaciones recientes en la escuela media (pp. 15-46). Buenos Aires, Argentina: Manantial.

Tiramonti, G., y Ziegler, S. (2008). La educación de las elites. Aspiraciones, estrategias y oportunidades. Buenos Aires, Argentina: Paidós.

Villa, A. (2012). Educación y destino en clave intergeneracional. En S. Ziegler y V. Gessaghi (Comps.), Formación de las elites. Investigaciones y debates en Argentina, Brasil y Francia (pp.85-103). Buenos Aires, Argentina: Manantial.

Ziegler, S. (2007). Los de excepción: un retrato de las elecciones escolares de las familias de sectores favorecidos en la Ciudad de Buenos Aires y el Conurbano Bonaerense. En M. Narodowsky y M. Gómez (Comps), Escuelas y Familias. Problemas de diversidad cultural y justicia social (pp. 7999). Buenos Aires, Argentina: Prometeo Libros, Educa.

Ziegler, S. (2014). Talleristas: la figura de los docentes extraordinarios. En G. Tiramonti (Comp.), Escuelas secundarias y talleres extracurriculares: otros sentidos de la experiencia escolar (pp. 21-26). Buenos Aires, Argentina: FLACSO.

Ziegler, S. (2015). Schools and families: school choices and formation of elites in present day Argentina. En J. Kenway y A. Koh Soon Lee (Eds.), Elite Schools: Multiple Geographies of Privilege (pp. 202-216). Londres, Inglaterra: Routledge. 
Ziegler, S. (2017). Personalization and Competition in Elite Schools in Buenos Aires: School Strategies for the Production and Legitimization of Dominant Groups, Journal of Education and Work, 30(2), 145-155.

Ziegler, S., Gessaghi, V., y Fuentes, S. (2018). Elite schools and institutional ethos: differential options and profiles in the education of privileged sectors in Argentina. En A. Van Zanten (Ed.), Elite Education. Major Themes on Education. Vol 2. Londres, Inglaterra: Routledge. 